\title{
La guerra enseñada: los conflictos bélicos en los manuales de lengua alemana elaborados en España antes y después de $1914^{1}$
}

\author{
Bernd MARIZZI \\ Universidad Complutense de Madrid \\ marizzi@filol.ucm.es
}

Recibido: 25 de noviembre de 2014

Aceptado: 21 de enero de 2015

\section{RESUMEN}

El presente artículo tiene como objetivo analizar el modo en el que la guerra y temas relacionados con los conflictos bélicos aparecen en los manuales y gramáticas de la lengua alemana hechos en España hasta los años 60 del siglo pasado. La premisa central de la que parte la investigación es que los manuales escolares constituyen uno de los elementos claves en la formación de identidades colectivas: en este sentido contribuyen a formar la imagen de Alemania que se tiene en España antes y después de la Gran Guerra. De esta manera la presencia de los temas militares en los materiales para la enseñanza de la lengua alemana refleja las relaciones de España con el mundo germanófono en momentos decisivos de la historia reciente.

Palabras clave: Alemán como lengua extranjera, libros de texto, identidades colectivas, temática bélica, transfer cultural Alemania-España.

\section{Teaching the War: Armed Conflicts in the German Language Manuals made in Spain before and after 1914}

\begin{abstract}
This article aims to analyse the way in which war and issues related to military conflicts are treated in manuals and grammars of the German language made in Spain until the sixties of the last century. The central premise which underlies the present research is that school textbooks are one of the key elements in the formation of collective identities: in this sense they contribute to the formation of the image of Germany in Spain before and after the Great War. In this way the presence of military issues in the German-language teaching materials illustrates the relations of Spain with the Germanspeaking world at crucial moments of recent history.
\end{abstract}

Keywords: German as a foreign language, Textbooks, Collective Identities, War, Cultural Transfer Germany-Spain.

SUMARIO: 1. Introducción. 2. Contacto lingüístico entre España y el mundo germanófono. 3. La guerra en libros de texto en distintos momentos de la historia. 3.1. 1870/71-1914. 3.2. Primera Guerra Mundial. 3.3. Guerra Civil Española. 3.4. Segunda Guerra Mundial. 4. Conclusión.

\footnotetext{
${ }^{1}$ El presente trabajo se inscribe dentro de las actividades del Proyecto de investigación "Las retóricas del clasicismo: los puntos de vista (contextos, premisas y mentalidades)" de la UCM, $\mathrm{n}^{\circ}$ ref. FFI2013-41410-P y del Grupo de investigación "RECEPTION - Estudios de recepción" de la Universidad de Alcalá, nº ref. CCHH2010/R24.
} 


\section{Introducción}

Los acontecimientos cuyo centenario se conmemoraron globalmente durante los últimos meses cambiaron el "mundo de ayer" y sentaron las bases del mundo globalizado en el que vivimos hoy en día. Conocer cuanto más detalles del "siglo corto" que fue el siglo XX aporta claves para enfrentarse a las vicisitudes de la actualidad y en este sentido los análisis de obras de texto y manuales escolares ayudan a comprender mejor las relaciones entre los distintos pueblos europeos.

Entre las múltiples razones que de siempre se aducen para adquirir un segundo idioma (exégesis de escritos fundamentales, comercio, comunicación con otras etnias) no ha faltado nunca la afirmación de que en el campo militar saber lenguas es de suma importancia. Es conocida la afirmación del lingüista francés Paul Levy (1950) de que en el s. XVI, en Francia, la lengua alemana se estudiaba sobre todo porque se consideraba útil en lo militar.

\section{Contacto lingüístico entre España y el mundo germanófono}

Ya desde la Baja Edad Media existían contactos lingüísticos entre España y los países de habla alemana, pero en la literatura reciente (Glück 2013: 501 y ss.) se señala que en el balance del aprendizaje de la lengua del otro se da un considerable desequilibrio. Se estudió mucho más español en el mundo germánico que alemán en España. La causa de ello reside en al menos dos hechos: el alemán tardó mucho en ser aceptado en el círculo de las lenguas de cultura importantes y la supremacía política del imperio español hacía muy atractivo su idioma. El francés ha sido tradicionalmente la primera lengua extranjera en España y en buena parte de Europa. En España, aparte de la innegable posición señera del francés como la lengua en la que se articulaban los avances culturales más significativos, también son responsables para el predominio de la lengua francesa la proximidad geográfica y el hecho de que los dos idiomas pertenecen a la misma familia de lenguas.

Los incentivos fundamentales para el aprendizaje de un idioma extranjero eran hasta bien entrado el siglo XVIII, sobre todo, los beneficios directos que se obtenían al poder comunicarse con hablantes de otros dominios lingüísticos.

Por los datos de que disponemos (Marizzi 2002 passim), tras la temprana aparición del Vocabulari molt profitos de 1502, hasta 1783 en España no se publicaron manuales que se pudieran considerar como testimonio impreso de un interés por el aprendizaje de la lengua alemana. Esto no significa, sin embargo, que en la Península no se aprendiera la lengua alemana, ya que en los fondos históricos de las bibliotecas españolas se encuentran, por un lado, diccionarios plurilingües, "Sprachbücher" y nomenclaturas español-alemán de los siglos XVII y XVIII'. Sus

${ }^{2}$ Cfr: Juan Angel de SUMARAn: Newes Sprachbuch (1623); Stephan BARnABÉ: Vnterweisung Der Spanischen Sprach (1657); Nicolás MEZ DE BRAIDENBACH: Gramatica, o instruccion española y alemana (1666); Christian Gottfried ReINHARDT: Der Spannisch Liebende Hochdeutscher (1696); 
autores fueron sobre todo españoles afincados en los países de habla alemana y, puesto que la mayoría de estas obras están configuradas de tal modo que se pueden utilizar tanto para aprender español como para aprender alemán, es muy posible que se utilizaran también en España. Y por otro lado, también se encuentran gramáticas alemanas redactadas o bien en francés, italiano e inglés o bien traducidas a estos idiomas.

Es muy posible que algunos de estos materiales hayan servido para la enseñanza del alemán y sabemos que varios de estos libros se utilizaron como fuente para la redacción de las primeras gramáticas alemanas hechas en España. Sin embargo, hay que tener siempre en cuenta que, hasta la primera mitad del s. XVIII, la lengua alemana no gozaba del mismo prestigio social frente al español y al francés, y no fue considerada como una lengua de utilidad práctica ni siquiera en los mismos territorios de habla románica dominados por los Habsburgo ${ }^{3}$.

La aplicación de nuevas tecnologías por mineros e ingenieros alemanes, por ejemplo, en las minas de mercurio y cinabrio en Almadén o de plata en Perú -para España de interés fundamental- no han dejado testimonio impreso que documente que en este ámbito se haya estudiado el alemán.

Desde finales de la Edad Media y especialmente, durante las guerras de gabinete, uno de los motivos para aprender lenguas extranjeras era el deseo de ser capaz de comunicarse con soldados de otras lenguas, porque la idea de un ejército nacional lingüísticamente uniforme sólo se implantó con la Revolución Francesa y tampoco se mantuvo en los ejércitos napoleónicos. Hasta entonces, los príncipes contaban para su ejército con mercenarios reclutados en regiones con excedentes entre los campesinos. En el caso de España, fueron los Lansquenetes (Guardia Alemana) de los Habsburgo y más tarde en la Guerra de Sucesión Española los Regimientos Suizos de los Borbones. Pero este contacto de lenguas en España no se reflejó en la producción tradicional de materiales de enseñanza del idioma alemán hasta los inicios del s. XX.

Las primeras gramáticas para el estudio de la lengua alemana en España datan de finales del siglo XVIII. Por un lado se trata de un manuscrito del año 1783 Gramática Española y Alemana. Esto es: Reglas que enseñan el leer, pronunciar, entender, y hablar el Idioma Alemán. Escrita por Fr. Raymundo Stráuch (sic), Religioso Menor Observante, que se conserva sin catalogar en la Hemeroteca del Archivo y Biblioteca Episcopal de Vic.

$\mathrm{Su}$ autor es el futuro obispo de Vic, Raymundo Strauch y Vidal (1760-1823), hijo de Franz Strauch, natural de Silesia y militar del Regimiento Betschart de la Guardia Suiza con destino en Tarragona (Marizzi 2012a: 96-99). Strauch y Vidal

Juan DE SotTOMAYOR: Llave capital (1706); A. F. K.: Teutsch-Spanischer und Regul=mäßiger Sprach=Zeiger (1712); Ernesto José EDER, Florilegio español y aleman o gramatica de la lengua alemana para los españoles, y española para los alemanes (1714); Antonio MORATORI: Instruccion fundamental (1723). Véanse referencias bibliográficas en WIPPICH-ROHÁKOVÁ 2000.

${ }^{3}$ Otra cosa ocurría en la parte de habla no germana de la monarquía donde la lengua alemana pronto se convirtió en lengua de cultura. 
fue durante un breve espacio de tiempo capellán del tercer Regimiento Suizo y, a pesar de que hoy en día está completamente olvidado, fue a principios del siglo pasado una de las principales figuras de la España ultraconservadora (Carlismo, Neocatolicismo, CEDA). Su olvido se debe sobre todo a su muerte violenta durante la ocupación de España por los Cien Mil Hijos de San Luis que puso fin al "Trienio Liberal" (1820-1823). A principios del s. XX hubo varios intentos de canonizarle.

Obviamente el modo de aprendizaje natural es el contacto directo con un germanohablante pero sobre esto disponemos de pocos datos respecto al mundo militar. Cierto es que el autor del manuscrito de la primera gramática alemana hecha en España, Raymundo Strauch, presume de haber aprendido alemán de "boca de su padre" pero en anteriores estudios (Marizzi 2012a: 98) demuestro que no fue así y que sus conocimientos de alemán no debieron ser muy profundos. Un caso curioso en este aspecto son las notas de Francisco Neto y Mesa (flor. 1802-1809), que también fue capellán de los Regimientos Suizos en Menorca y Madrid a principios del s. XIX. De estas notas, conservadas en la Biblioteca Pública de Mahón (MSS 77), se sabe que Francisco Neto tomó clases en 1802 y1803 tanto con un sargento del Regimiento Suizo llamado Andrés Bacher como con otro cabo de nombre Elias del mismo regimiento y que luego contrató a un criado germanófono de nombre Sucher para practicar el alemán. Por otro lado, entre los fondos de Mahón se encuentra bajo la signatura 11318 un ejemplar anotado de la Gramática alemana de Villa de 1792 que a continuación se menciona. Estas notas son del mismo capellán militar Neto y Mesa e indican que estudió, utilizó, analizó y comentó la primera gramática alemana editada en España.

En 1792 apareció, pues, en Madrid la primera gramática impresa para la enseñanza del alemán, la Gramática de la lengua alemana de Antonio de Villa (flor. 1788-1811). El autor de esta gramática también fue religioso, en este caso Dominico y Cura de Extranjeros en el Hospital General de Madrid. Las dos primeras gramáticas de alemán en España se relacionan, por lo tanto, con el mundo religioso y ambas se basan en un original en francés. La de Strauch (1783) es la versión española de C. Leopold (1690) y la de Villa (1792) se basa en una adaptación francesa de la Grundlegung der deutschen Sprachkunst (Leipzig, 1725) de Johann Christoph Gottsched (1700-1766), un hecho muy común en la producción cultural en la España de ese tiempo ( $c f r$. Strauch: Marizzi 2012a: 100 y Villa: Marizzi 2012b: 135-137) y que perdura hasta bien entrado el siglo XX.

Desde la primera mitad del s. XIX aumentan los contactos directos con la cultura en lengua alemana como intento de una parte de la nueva burguesía liberal de liberarse de los antiguos poderes fácticos. La ley Pidal de 1845 introduce las lenguas vivas francés, inglés y alemán en el currículum de las enseñanzas medias y la importación del Krausismo por Sanz del Río pone las bases para la renovación de la vida intelectual que culmina con la Institución Libre de Enseñanza. Todo ello redunda en que durante más de un siglo la lengua alemana adquiera en España un gran prestigio como lengua de la ciencia y de la filosofía ( $c f r$. Marizzi 2012c: 491-495). Este acer- 
camiento a la cultura en lengua alemana ciertamente no está desprovisto de ciertas zonas oscuras como el acercamiento que hubo entre el franquismo y el nazismo.

Muestra de este nuevo interés por la lengua alemana es el considerable aumento en España de la producción de métodos para estudiar alemán durante estos años. Esta producción autóctona se mantuvo hasta que alrededor de 1960 - 1965 se empezaron a utilizar en España cada vez más manuales hechos en la República Federal de Alemania $^{4}$.

\section{La guerra en materiales de aprendizaje de lengua alemana en distintos momentos de la historia}

En los métodos anteriores a 1870/71 las referencias al mundo militar están presentes en algunos manuales de lengua alemana analizados.

Algunas gramáticas y "Gesprächsbücher" incluyen nomenclaturas y entre ellos cabe destacar, aunque se sitúe fuera del ámbito lingüístico castellano, el Vocabulari molt profitos que en 1502 publicó Hans Rosembach en Perpiñán que entonces pertenecía a la Corona de Aragón (Facsímil en Stegmann 1991). Dedica dos capítulos de unas 4 páginas en total al mundo bélico y en el f. 34r dice que "Lo .xxiiii. c. es de armes y mestre de darmes - Das .xxiiii. capitel ist von den gewaffen und von wafner" y, más adelante, en el f. 43v "Lo .xxxviii. ca. es de batalla y guerra y jugadors - Das xxxviii. ca. ist von stryten und kriegen und spiler" (Stegmann 1991: 67 y ss. y 86 y ss. respectivamente).

Avanzando cronológicamente se ha de subrayar que la primera gramática alemana, la de Strauch (1783) se basa en un modelo francés de temática militar y de ahí la razón por la cual se dan muchos ejemplos sueltos del campo que aquí interesa "der in der gefähr verständige und unerschrokene hauptmann, el capitan prudente é intrepido en el peligro", (f. 30v) y "Die churfürsten schicken dem Kayser ihre Völcker nicht, Los Electores no envían sus Tropas al Emperador" (f. 43).

En la primera gramática alemana impresa en España, la Gramática de la lengua alemana de Antonio de Villa (flor. 1788-1811) no hay ningún epígrafe especial dedicado a la guerra, pero el apartado "ciudad y sus partes" incluye los elementos defensivos y el término "Geschütz" (Villa 1792: 373 y ss.).

El último manual que incluye este tipo de repertorios es Casey 1846 que en el apartado de la ciudad y sus partes habla de "elementos defensivos" (Casey 1846: 167) y más adelante "De los términos de la guerra" (Casey 1846: 180 y ss.), sin incluir ningún término naval.

\footnotetext{
${ }^{4}$ En un futuro no muy lejano esperamos presentar una completa bibliografía de los manuales de enseñanza del alemán en la península ibérica.
} 
En el Curso de literatura de la muy voluminosa gramática de dos tomos de Fernández de Castroverde 1867/68 se incluyen lecturas que tratan de batallas importantes. Dos de ellas, "Die Schlacht bei Lützen im Jahre 1632" (Fernández 1867: 126-133) y "Magdeburgs Zerstörung" (Fernández 1867: 139-144) proceden de la Geschichte des dreißigjährigen Kriegs (1790) que Friedrich Schiller escribió en Jena cuando aún quería ser Universalhistoriker.

\subsection{0/71-1914}

A partir de 1870/71 el ejército prusiano que había vencido en Sedan se convirtió en punto de referencia para el reformismo militar español del último tercio del siglo XIX. Esta afinidad en lo militar tiene su punto más palpable en el hecho de que se introdujera la enseñanza del alemán en el currículum de las Academias Militares de Ávila y Toledo a finales del XIX. En cuanto a los materiales de enseñanza cabe destacar las obras Deutsche Sprachübungen (1900) y la "Gramática Alemana Militar" (1905/06) en dos volúmenes que publicó Cesáreo Olavarría (1863-1947). Este militar fue profesor de esta lengua en las mencionadas Academias y también en la antigua Escuela Central de Idiomas de Madrid. Los Deutsche Sprachübungen de hecho constituyen la única gramática alemana conocida que está impresa en letras manuscritas alemanas ${ }^{5}$. En cuanto a ejemplos, situaciones, diálogos y lecturas, tanto los Deutsche Sprachübungen como la Gramática Alemana Militar se centran exclusivamente en la temática militar.

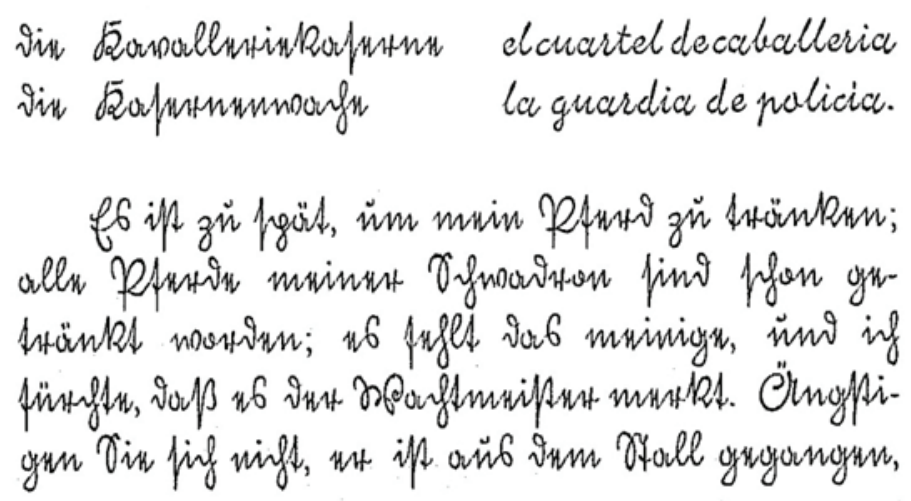

Figura 1: Olavarría 1900: 30 [Fuente: Colección B. Marizzi]

\footnotetext{
${ }^{5}$ Es lógico que la impresión de esta obra tuviera lugar en una empresa especializada alemana, la de Friedrich August Enpel en Sondershausen (Turingia). Además, los problemas de imprimir en España en Fraktur (MARIZZI 2012b: 141), la expansiva política comercial de editoriales alemanas como Brockhaus y el reclamo publicitario de "Impreso en Leipzig" llevó a varias editoriales españolas a encargar ediciones en Alemania (cfr. al respecto CEBALlos 2009: passim).
} 
La figura de César Olavarría puede servir de ilustración al militarismo reinante en el franquismo y en este contexto conviene sacar a colación que en marzo de 1939 presentó una versión reformada de su Gramática Alemana Militar $(1905 / 06)$ a la comisión dictaminadora de Libros de texto que los golpistas vencedores habían establecido para controlar el material de enseñanza. El único cambio que realizó Olavarría para que se pudiera utilizar en los Institutos Nacionales de Bachillerato el manual pensado para militares fue sustituir cualquier palabra relacionada con el mundo militar por otra civil.

Aunque no llegara a producir cambios sustanciales en la organización del ejército español, sí se denota en las obras analizadas la importancia que se concede al Imperio Alemán en general y a sus protagonistas Guillermo II y Bismarck como artífices de la unificación alemana.

Ejemplos de ello se encuentran en las Nuevas versiones alemanas de Manuel del Pino que incluye textos como "Fürst Bismarck" (Pino 1907: 119) y "Der Kaisertag zu Versailles" donde se dice:

In dem Schlosse Ludwig XIV, jenes gottlosen französischen Fürsten, dessen ganzes Sinnen und Trachten auf Deutschlands Zersplitterung und Erniedrigung gerichtet war, ward am 18. Januar 1871 durch eine feierliche Handlung König Wilhelm als deutscher Kaiser [la cursiva es del original, B.M.] ausgerufen (Pino 1907: 173).

En el manual Gramática y Ejercicios prácticos de Alemán de Ricardo RattiKámeke de 1916 el primer contacto de los alumnos con la lengua alemana y su fonética lo constituye un ejercicio de pronunciación que se sirve de aforismos de Guillermo II (Ratti 1916: XI) ${ }^{6}$. Esta veneración de las figuras claves del militarismo alemán se mantiene en el Florilegio Graduado (1928) de Domingo Sánchez Hernández con las "Frases célebres del Canciller de Hierro Príncipe Otto von Bismarck" que se extienden por nada menos que doce páginas (Sánchez 1928: 2637) y se inician con una frase que a los carlistas españoles tenía que sonar: "Ich glaube Gott zu gehorchen, wenn ich dem Könige diene mit der Devise: Mit Gott für König und Vaterland".

Aun en una obra de 1950 -Mein deutsches Buch de Rafael Rodríguez Estellés y Rafael Rodríguez Lapuente- sigue esta veneración con una alabanza del Canciller de Hierro (Rodríguez Estellés/Rodríguez Lapuente 1950: 153 y ss.).

La admiración que suscita la potencia militar alemana y su proyección colonial aparece en una gramática de 1914, la Antología Alemana del jesuita Antonio Guasch. Entre sus páginas se recoge un episodio de la guerra colonial alemana en Deutsch-Südwestafrika, hoy Namibia, en la que el Reich reprimió la sublevación de varias tribus africanas (Guasch 1914: 79). El texto describe la muerte de un oficial alemán en la lucha contra los que despectivamente son llamados hotentotes.

\footnotetext{
${ }^{6}$ Es interesante que en la segunda edición de este manual de 1935, que prácticamente es una reimpresión, lo único que falta es justamente este texto. Las circunstancias políticas habían cambiado.
} 
El posterior exterminio de los herero en los primeros campos de concentración y exterminio alemanes en un país extranjero obviamente no forma parte del relato.

\subsection{Primera Guerra Mundial}

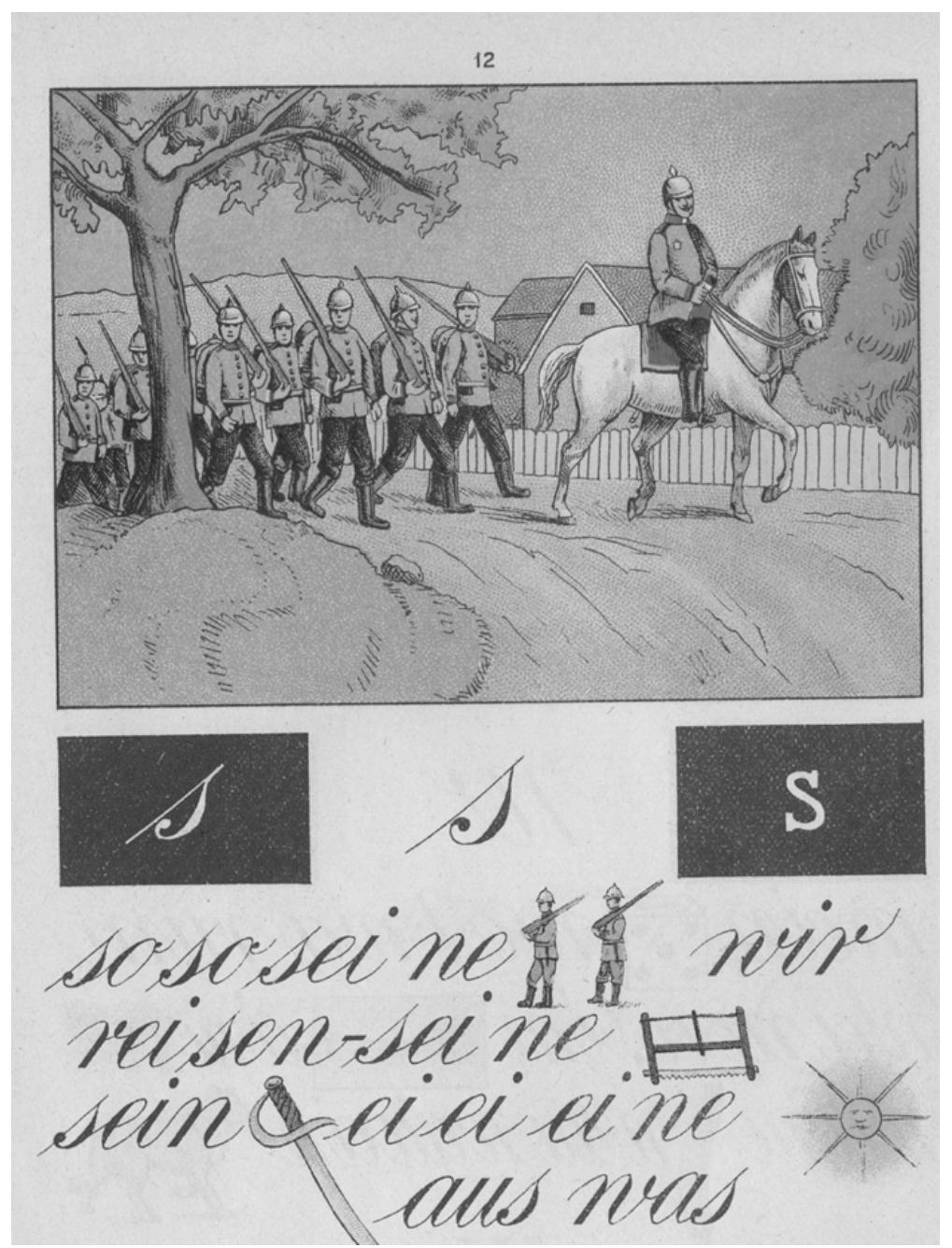

Figura 2: Koethke 1916 [Fuente: Colección B. Marizzi]

En la Primera Guerra Mundial la posición de España oficialmente fue neutral aunque no faltan voces que afirman que la neutralidad se impuso por la falta de potencial militar y las perspectivas tanto económicas como políticas. De hecho España vendía material bélico a ambos contendientes. 
Como primera presencia gráfica de la deflagración véase la imagen (figura 2) de soldados con la "Pickelhaube" que se incluye en la cartilla de lectoescritura de Wilhelm Koethke editada en 1916 por el Colegio Alemán de Málaga.

Durante estos años también se publicaron en Barcelona los dos primeros volúmenes de la Biblioteca Ratti para el estudio del alemán, la ya mencionada Gramática y ejercicios prácticos de alemán de Ricardo Ratti-Kámeke (Berlín 1873 Barcelona, 1945). En la primera de ellas (Ratti 1916) hay poca presencia de lo que nos interesa aquí, el mundo aún no había cambiado: Alemania sigue teniendo colonias (Ratti 1916: 75) y las conversaciones tratan de cosas que la guerra ha hecho imposible como un viaje de Múnich a Venecia en tren (Ratti 1916: 95) o que han cambiado radicalmente como el servicio militar (Ratti 1916: 124).

Este cambio radical que produjo el enfrentamiento entre los "sonámbulos" (Clark 2014) se pone de manifiesto, con el lógico retraso, en el segundo volumen (Ratti 1917). Aumentan considerablemente las referencias a la guerra como ocurre en la siguiente conversación: "Ist heute viel Neues vom Kriegsschauplatz gemeldet worden? - Se anuncia (es wird gemeldet) que la ciudad [de] Bukarest ha sido ocupada por nuestras tropas" (Ratti 1917: 62). Se está refiriendo a la ocupación de la capital rumana por un ejército multinacional bajo el mando alemán en diciembre de 1916 que se presenta como "nuestras tropas". En otra conversación se habla de la marina de guerra alemana (Ratti 1917: 74). En las frases sueltas aparece el tono épico que tradicionalmente necesita toda literatura bélica: "Wer trug die Fahne des Regiments? Ein Unteroffizier trug sie zuerst, und nach dessen Tod hat ein gewöhnlicher Soldat sie bis zum Ende des Kampfes getragen" (Ratti 1917: 90). Pero también se humaniza la narración: "Als der Wagen mit der Post aus der Heimat im Lager eintraf (ankam), umringten ihn die Soldaten erwartungsvoll, und jeder hoffte Nachrichten von den Seinen zu erhalten" (Ratti 1917: 196).

De todo ello se deduce que Ratti concibió el primer volumen aún en tiempos de paz mientras que en el segundo ya se refleja la realidad de la Primera Guerra Mundial tal y como la percibía el autor.

La guerra sigue presente en las Nociones elementales y prácticas de Lengua Alemana. En este manual manuscrito y hectografiado de Faustino Gosalbo y Mas de 1920 los ejemplos para los nombres compuestos en buena parte pertenecen al vocabulario bélico:

Torpedobootszerstörer, Unterseeboote, Scharzimmerflotte (sic), Maschinengewehre, Kriegsschauplatz, Feuertätigkeit, Feuerüberfall, Gefechstätigkeit (sic), Unterseebootkrieg, Konstitutionsversammlung, Witterungsverhältnisse, Schiffsverkehr, Minenwerfer, Schützengraben, Aufklärungsabteilung, Mittelstandskükken (sic) Nahrungsmittel, Kettenhanddelprozesse (sic), Kriegsrationierung (Gosalbo 1920: 16).

En ninguno de los libros de texto de esta época se encuentran reflejos del enfrentamiento entre germanófilos y aliadófilos que se produjo en la opinión pública 
española durante los años de la Primera Guerra Mundial ${ }^{7}$ y que se mantuvo hasta el franquismo.

La Primera Guerra Mundial también está muy presente en el primer curso de la Gramática Alemana de Francesc de Borja Moll que se publicó en 1938. Hay un claro posicionamiento a favor de la causa alemana - en los temas que hay que traducir al español "nuestros enemigos" son Rusia e Inglaterra:

12. Im östlichen Kriegsschauplatz nahmen wir den Russen sechshundert Mann, drei Geschütze und neuen Maschinengewehre ab. 13. Ein erneuter Durchbruchsversuch des Feindes endigte mit der Vernichtung seiner drei besten Regimenter. 14. Im heutigen Kampfe blieben auch unsere Truppen erfolgreich; der Feind liess sechs Offiziere, neunhundert Mann und acht Maschinengewehre in unseren Händen (Moll 1938: 213).

Los pasajes que tratan la Primera Guerra Mundial están situados en las últimas páginas de las dos ediciones del primer curso del manual que se han analizado para este artículo, la primera de 1938 y la segunda de 1939. Las dos ediciones son casi idénticas, solamente que en el año 1939 el colofón del volumen es "Die Wacht am Rhein" de Schneckenburger (Moll 2 1939: 235) mientras que en 1938 todavía figuraba "Der gute Kamerad" de Uhland (Moll 1938: 215). Cabe destacar que el cambio de una canción "sentimental" por otra más "revanchista" puede entenderse como un elemento añadido que persiguió crear o aumentar -en consonancia con la alineación del Régimen franquista y coincidiendo con el inicio de la Segunda Guerra Mundial- el sentimiento militarista y pro-alemán en la opinión pública española.

\subsection{Guerra Civil Española}

En el contexto de la Guerra Civil Española no se puede pasar por alto al autor que acabamos de citar: Francesc de Borja Moll [Casanovas] (1903-1991). Hoy en día es considerado, con razón, uno de los más importantes filólogos catalanes e incluso hay calles, colegios e institutos baleares que llevan su nombre. Hasta 2014 existía en Palma de Mallorca la Editorial Moll que fue un referente en la lucha por el catalán. El hecho de que en los primeros años del franquismo la represión y el control sobre los libros de texto en las Islas Baleares tuvieran una especial crueldad y virulencia puede, hasta cierto punto, explicar por qué en los manuales de alemán de esta época abundan pasajes bélicos que concuerdan con la orientación pro-alemana del Régimen. Este control se institucionalizó a partir de 1938 en la "Comisión Dictaminadora de Libros de Texto" y puede explicar lo que, desde nuestra actual perspectiva, resulta chocante: los temas para traducir al alemán que se encuentran en manuales de un autor que no es conocido por su pasado franquista parecen estar sacados de un parte de guerra de los sublevados.

\footnotetext{
${ }^{7}$ Sobre la imagen de Alemania en la prensa española de la época véase Ortiz de Urbina (2010).
} 
En el caso del ya citado primer curso de la Gramática Alemana de F. de B. Moll se dice en 1938:

1. Las tropas enemigas han atacado las fortificaciones nacionales. [...] 7. Esta mañana las fuerzas nacionales han proseguido su victorioso avance sobre el frente occidental. 8. Después de una sangrienta lucha han ocupado una importante posición que domina la carretera que va de Madrid a Albacete (Moll ${ }^{1}$ 1938: 214).

También en el segundo curso de su Gramática de 1939 aparecen referencias explícitas a la guerra española - "19. La batalla del Ebro fue decisiva en la guerra civil española" (Moll ${ }^{1}$ 1939: 230) - recogiendo la propaganda y falsificación de la historia por parte del bando ganador - "25. Los insurrectos han depuesto las armas y se han entregado a los soldados leales. [...] 28. Los rebeldes tenían en contra suya a todo el ejército" (Moll ${ }^{1}$ 1939: 77).

En ediciones posteriores, estos textos referidos a la Guerra Civil Española desaparecen mientras que el resto del manual no sufre cambios significativos ${ }^{8}$.

\subsection{Segunda Guerra Mundial}

Mientras que las referencias a la guerra civil española solamente aparecen en las gramáticas de Moll, la Segunda Guerra Mundial aparece en varios métodos concebidos para la enseñanza del alemán.

Uno de estos manuales de lengua alemana donde se muestra la filiación declaradamente franquista y filonazi es el Nuevo método práctico de Lengua Alemana del que se ha podido analizar la segunda edición de 1941 y la sexta de 1950 . Su autor fue el ya mencionado Domingo Sánchez Hernández (1896-1981), alumno del Pedagogium español de Paz de Borbón (1862-1946) en Múnich, censor y traductor de Franco y durante muchos años catedrático de alemán en el Instituto de Enseñanzas Medias Ramiro de Maeztu. El prologuista de la gramática, Félix Díez Mateo, también censor en Salamanca y luego profesor de alemán en el Instituto de Bilbao, describe el alemán como:

[...] la lengua del pueblo que fue sumida a la esclavitud odiosa impuesta por el tratado de Versailles, esclavitud de la que ha sabido redimirse [...] La lengua del pueblo que con Italia nos ayudó en nuestra guerra contra el marxismo (pues, a no ser por esas buenas amistades, se habría roto en muchos pedazos nuestra España y habría dejado de ser católica) (Sánchez $\left.{ }^{2} 1941: 5\right)$.

El ilustrador, Fernando Villalobos Mier (1919-1990), luchó en el bando sublevado en el frente de Lérida. En el texto del manual no abundan las referencias a la

\footnotetext{
${ }^{8}$ Con el tiempo Moll fundó la Editorial Moll que fue un referente en el ámbito balear y catalán abarcando sus publicaciones una gran variedad de temas e idiomas extranjeros. Junto con Moll son varios los autores que publicaron manuales de distintas lenguas en varios momentos de la historia de España, entre ellos por citar algunos que tienen que ver con el alemán: Francisco García Ayuso (1835-1897), Ricardo Ratti-Kámeke (1873-1945), Enrique Kucera (1905-1959) y Johann Rauter Schurian (flor. 1941-1970).
} 
guerra, solo un pasaje que habla de la "Kriegswirtschaft" (Sánchez ${ }^{2}$ 1941: 161). Sin embargo abundan los pasajes en los que se manifiestan las simpatías del autor y de las autoridades que aprobaron el manual para su uso en los institutos. El manual incluye un discurso de Hitler (Sánchez ${ }^{2}$ 1941: 137) y la reproducción de una carta de Hitler dirigida a Sánchez Hernández en la que éste le da las gracias por la felicitación que el "Herr Professor" y sus alumnos del Instituto de Salamanca le habían dirigido por la "Wiedervereinigung" de Austria con el Reich en 1938 (Sánchez ${ }^{2}$ 1941: 210).

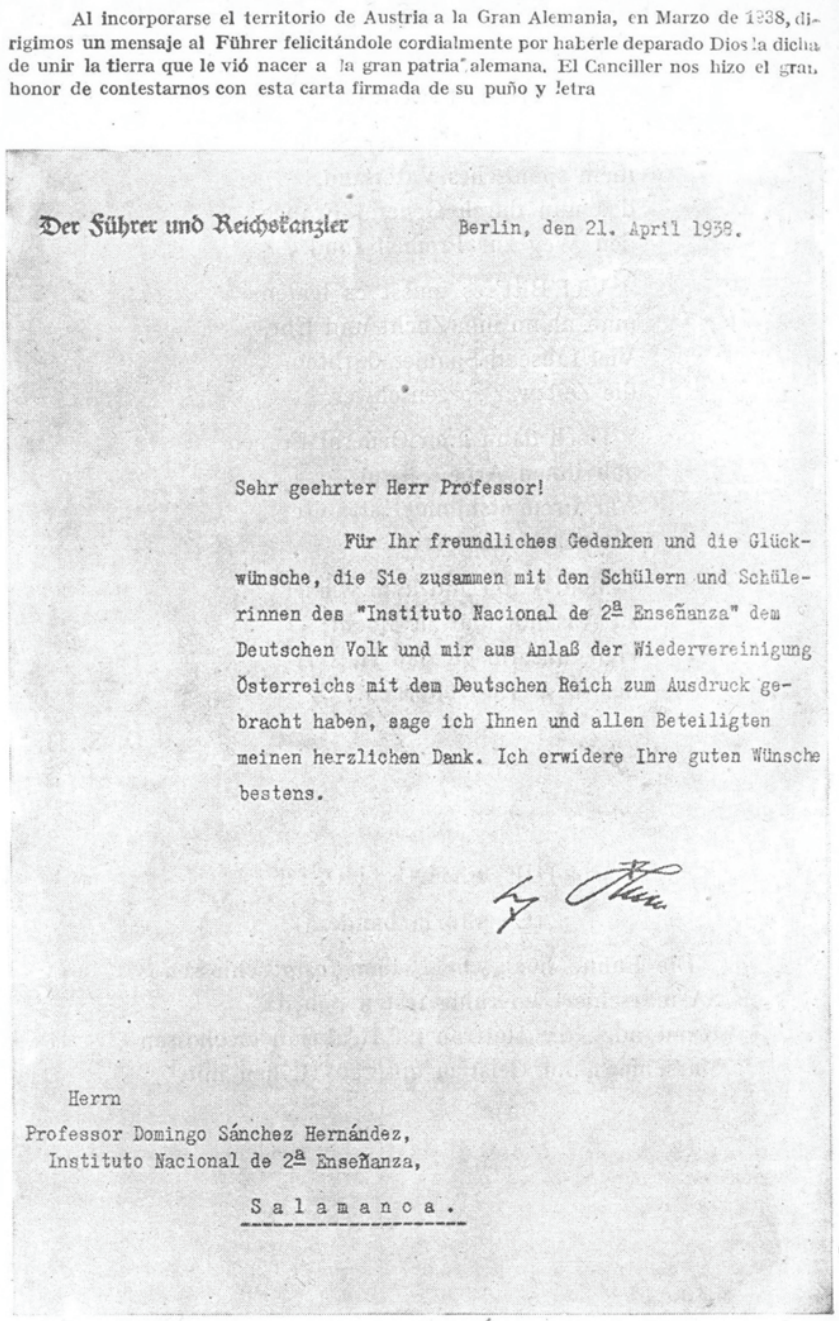

Figura 3: Sánchez ${ }^{2}$ 1941: 210 [Fuente: Colección B. Marizzi] 
En la segunda edición de 1941 y anterior a esta carta se encuentra una poesía en alemán en la que Sánchez Hernández alaba a Franco:

Heil Franco

Ich liebe mit ganzer Seele

mein spanisches Vaterland, das nun durch General Franco

den Weg zur Freiheit fand (Sánchez ${ }^{2}$ 1941: 209).

y el himno del partido nazi, el "Horst-Wessel-Lied". Estos fragmentos faltan en la $6^{\text {a }}$ edición de 1950.

En la primera edición de la primera parte de la Gramática Alemana de José Weinberger, publicada en 1941, la guerra aún no existe: en la lección 22, por citar un ejemplo, se habla de la geografía de Europa sin más y se aprende cómo redactar una carta para pedir trabajo (Weinberger 1941: 178). También en otras muchas situaciones el ambiente que se percibe es el de los tiempos de paz. En el prólogo, sin embargo, el autor formula el deseo de:

¡Que esta Gramática estreche cada vez más los lazos científicos y culturales de dos pueblos tan unidos a través de la Historia, y más unidos en la actualidad con lazos de sangre en la lucha contra el comunismo! Con la esperanza e íntimo deseo de ver realizadas estas aspiraciones entrega a la publicidad su modesta obra (Weinberger 1941: VI).

Pero en la segunda parte de este manual del año 1942 Weinberger aumenta considerablemente los ejemplos que tienen que ver con la guerra: Varias lecturas tratan de "Die Kanonenfabrik von Krupp" (Weinberger 1942: 138-142-147) y en un texto ("Der Fallschirm als Lebensretter") sobre el uso de los paracaídas en la Primera Guerra Mundial se ensalza al aviador de combate de esta guerra Ernst Udet (1896-1941), una de las figuras preferidas de la propaganda nacionalsocialista.

Im letzten Weltkrieg rettete der Fallschirm vielen Fliegern und Ballonbeobachtern das Leben. Generalmayor Udets Flugzeug erhielt eines Tages von einem französischen Jagdflieger Schüsse in den Benzintank. Das Flugzeug begann zu brennen. Udet sprang aus einer Höhe von 80 Metern über dem Erdboden ab. Er landete im Trommelfeuer der feindlichen Maschinengewehre, eilte mitten durchs Feuer auf die deutschen Schützengräben zu und war gerettet (Weinberger 1942: 201 y ss.).

También en los ejercicios acerca de oraciones temporales se recurre a imágenes propagandísticas " 6 . Während die Soldaten draußen an der Front kämpfen, arbeiten die Volksgenossen in den Fabriken, um die nötigen Waffen herzustellen" y en el glosario se ofrece la acepción de la expresión nacionalsocialista "der Volksgenosse" como "el compatriota”. (Weinberger 1942: 276)

Quedan por comentar las obras del jesuita Teodoro Baumann (1907-1992). En los tres volúmenes de su Werkbuch der deutschen Sprache de 1943 aparecen va- 
rias referencias al temario bélico. El más extenso (dos páginas) es una lectura ("Pioniere zerschlagen Sowjetbefestigungen") al estilo de los cómics "Hazañas bélicas" dónde heroicos zapadores alemanes luchan contra los "rojos":

Seit zwölf Stunden hocken die Pioniere in ihren Erdlöchern. Aus den Bunkern knattern die MGs. Nebelschwaden hindern die Sicht. Doch bald ändern sich die Verhältnisse. [...] Die Roten stellen das Feuer ein. [...] Mit Handgranaten und Maschinenpistolen wird die Besatzung, die sich nicht ergeben will, erledigt (Baumann 1943b: 10).

Intervienen "nuestros" aviadores y al final "Die vielen rauchenden Trümmer vernichteter russischer Flugzeuge beweisen den deutschen Sieg" (Baumann 1943b: ibid). Si en la parte práctica de este método Baumann recurre a narraciones que pretenden captar el interés de un público afín a los vencedores y sus aliados, en la parte teórica se ilustra la formación de compuestos alemanes con "Obersturmbannführersgattin" (Baumann 1943c: 12).

La unión entre los dos regímenes se percibe claramente en la versión española de la frase: "Der Führer selbst überreichte dem General den Orden" que no es otra que "El caudillo mismo (en persona) entregó la condecoración al general" (Baumann 1943a: 21).

La seguridad de Baumann sobre la continuidad del III Reich se pone de manifiesto en el hecho de que la edición de 1943 de su gramática incluye una carta comercial con el saludo "Heil Hitler" que lleva fecha de agosto de 1945 (Baumann 1943b: 102).

\section{Conclusión}

A pesar de una aparente impermeabilidad o neutralidad ideológica, los manuales y métodos destinados a la enseñanza de una lengua, al igual que cualquier otra forma de producción intelectual, permiten al investigador un análisis del discurso ideológico que su autor está trasmitiendo, de manera consciente o inconsciente, ya sea sincero o acomodado a presiones externas.

Los ejemplos, en tanto que ilustraciones de reglas gramaticales y por su capacidad para transmitir contenidos, conforman un terreno privilegiado para filtrar lo que podríamos denominar premisas discursivas, las cuales, en este particular ámbito, abarcan desde información relativa a la actitud y mentalidad del autor hasta una determinada presentación del interés otorgado al aprendizaje de una lengua particular (Perelman /Olbrechts-Tyteca 1994: 536 y ss.). Ese es el caso del sesgo militarista que se asociaba a la lengua alemana, espoleado por un período histórico, desde finales del siglo XIX hasta mediados del XX, en que los países de lengua alemana han mantenido un triste protagonismo en los conflictos bélicos que han tenido lugar en el continente europeo.

Los ejemplos aportados por las gramáticas de alemán para estudiantes españoles o hispanohablantes nos permiten, de ese modo, seguir un hilo histórico de la 
ideología de sus creadores, en su mayoría nítidamente conservadora, y del ambiente político en que se desenvolvía su labor. Podemos así observar cómo la presencia de temas militares aumenta considerablemente con el establecimiento del Imperio Alemán como potencia militar en el panorama internacional a partir de la Guerra franco-prusiana. Durante la Primera Guerra Mundial la escisión de la opinión pública española entre germanófilos y aliadófilos no repercute en la imagen de Alemania como potencia militar transmitida por los libros de texto de lengua alemana. La afinidad política e ideológica entre la España franquista y la Alemania nazi deja un buen número de referencias en los manuales analizados, en los cuales podría hablarse abiertamente de propaganda política.

Por otro lado, un determinado silencio puede ser tan elocuente como una manifestación expresa: el déficit de voces críticas queda patente en la ausencia de ejemplos de naturaleza antibelicista o pacifista en estos manuales.

\section{Referencias bibliográficas}

ARXIU I BIBLIOTECA EPISCOPAL DE VIC: Strauch R., Gramática Española y Alemana. Esto es: Reglas que enseñan el leer, pronunciar, entender, y hablar el Idioma Alemán. Escrita por Fr. Raymundo Stráuch [sic], Religioso Menor Observante. Año 1783. Ms. sin catalogar.

Baumann, T., Werkbuch der deutschen Sprache. II. Kurs. Formenlehre, a) Regelbuch. Cádiz/Madrid: Escelicer 1943a.

BAUMAnN, T., Werkbuch der deutschen Sprache. II. Kurs. Formenlehre, b) Übungsbuch. Cádiz/Madrid: Escelicer 1943b.

Baumann, T., Werkbuch der deutschen Sprache. III. Kurs. Wort- und Satzlehre. Cádiz/Madrid: Escelicer 1943c.

Biblioteca PÚblica de MahÓN. Notas manuscritas de Francisco Neto y Mesa, 8 h. (MSS 77).

CASEY, G., Nueva gramática teórica y práctica de la lengua alemana. Barcelona: José Tauló 1846.

Ceballos Viro, Á., Ediciones alemanas en español (1850-1900). Madrid/Frankfurt am Main: Iberoamericana/Vervuert 2009.

Clark, C., Sonámbulos: cómo Europa fue a la guerra en 1914. Barcelona: Galaxia Gutenberg, Círculo de Lectores 2014.

DEL PINO [GonZÁLEZ], M, Nuevas versiones alemanas. Madrid: Ratés 1907.

FERnÁndeZ De CASTROverde, C., Gramática Alemana. Barcelona: s.n. [Leipzig: en la imp. de F.A. Brockhaus] 1867/68.

GLÜCK, H., Die Fremdsprache Deutsch im Zeitalter der Aufklärung, der Klassik und der Romantik. Wiesbaden: Harrassowitz 2013.

Gosalbo y Mas, F., Nociones elementales y prácticas de Lengua Alemana. Barcelona: s.n. [Librería de Adustin Bosch] 1920.

GotTSCHED, J. C., Grundlegung der deutschen Sprachkunst. Leipzig: Breitkopf 1725, ${ }^{5} 1762$.

GuASCh [I Bufí], A., Antología Alemana. Barcelona: s.n. [Tip. Católica] 1914.

KoethKe, W., Fibel für Deutsche Auslandsschulen. Málaga: s.n. [Alcalá Buch- und Steindruckerei] 1916.

LEOPOLD C., L'art de parler allemand. París, Viena: s.n. 1690, 1728, 1744, 1745, 1761. 
LeVy, P., La langue allemande en France. Pénétration et diffusion des origines a nos jours. París, Lyon: IAC 1950.

MARIZZI, B., Historia de los métodos de enseñanza del alemán en España. Santiago de Compostela: Asociación Galega de Xermanistas 2002.

MARIZZI, B.. «Observaciones sobre la Gramática Española y Alemana de Raymundo Strauch y Vidal de 1783», en: PfEIFFER M. / MONTANER, A. / VINARDELL, T. (eds.), Was mich wirklich interessiert. Homenatge a Jordi Jané. Girona: Documenta Universitaria 2012a, 95 - 108.

MARIZZI, B., "La Gramática de la lengua alemana de Antonio de Villa (1792): fuentes y correctores», Revista española de lingüística aplicada (RESLA), 25 (2012b), 133-145.

MARIZZI, B., «Deutsch als Wissenschaftssprache (DaW) in den Lehrwerken des Deutschen für Spanier von Richard Ratti-Kámeke zwischen 1916 und 1943», en HESS-LÜTTICH, E.W.B. et. al (eds.), Re-Visionen. Frankfurt am Main: Lang 2012c, 491-511.

Moll [Casanova], F. de B., Elementos de Gramática Alemana, primer curso. Palma de Mallorca: Mossén Alcover ${ }^{1} 1938,{ }^{2} 1939,{ }^{4} 1941$.

Moll [CASAnova], F. de B.: Gramática Alemana, segundo curso. Palma de Mallorca: Mossén Alcover ${ }^{1} 1939,{ }^{2} 1943$.

Olavarría MARTíneZ, C., Ejercicios de Idioma alemán - Deutsche Sprachübungen. Madrid: Romo y Füssel [Sondershausen: Fr. Aug. Enpel,] 1900.

Olavarría MartíneZ, C., Gramática Alemana Militar. Toledo: s.n. [Imprenta, escuela tipográfica y encuadernación del colegio María Cristina] 1905/06.

ORTIZ DE URBINA SOBRINO, P., «La imagen de Alemania en España (1860-1920). Resultados de un análisis hemerográfico», Revista de Filología Alemana, Anejo II (2010), 277 - 287.

Perelman, Ch. / L. OlBRECHTS-TYTECA, Tratado de la argumentación. La nueva retórica. Madrid: Gredos 1994.

RAtTi-KÁMeKe, R.: Gramática y Ejercicios prácticos de Alemán. Primera Parte. Barcelona: s.n. [Modesto Bertós] 1916.

RATTI-KÁMEKe, R.: Gramática y Ejercicios prácticos de Alemán. Segunda Parte. Barcelona: s.n. [Modesto Bertós] 1917.

RodríGuez Estellés, R. / R. RodríGuez LAPUente, Mein deutsches Buch. Valencia: s.n. [Sucesor de Vives Mora] 1950.

SÁnCHEz HernándeZ, D., Florilegio Graduado para la Enseñanza del Alemán. Madrid: Sucesores de Rivadeneyra 1928.

SÁnCHEZ HERnÁNDEZ, D., Nuevo método práctico de Lengua Alemana. Bilbao: s.n. [Gráficas Grijelmo] ${ }^{1} 1940,{ }^{2} 1941,{ }^{6} 1950$.

Stegmann, T. D., Vocabulari Català-Alemany de l'any 1502 = Katalanisch-deutsches Vokabular aus dem Jahre 1502. - Nachdruck der von Pere Barnils besorgten Faks.Ausgabe. Frankfurt am Main: Domus Ed. Europeae 1991.

VILLA, A., Gramática de la lengua alemana. Madrid: Imprenta Nacional 1792.

Weinberger, J., Gramática Alemana. Primera parte. Morfología. Madrid: Nueva Época ${ }^{1} 1941,{ }^{2} 1943$.

WeInBerger, J., Gramática Alemana. Segunda parte. Sintaxis. Madrid: Nueva Época 1942.

WIPPICH-ROHÁKOVÁ, K., Der Spannisch Liebende Hochdeutscher: Spanischgrammatiken in Deutschland im 17. und frühen 18. Jahrhundert. Hamburgo: Buske 2000. 